\title{
SALUD MENTAL, EXPERIENCIAS NEGATIVAS EN LA INFANCIA Y SU IMPACTO SOBRE LA EXPERIENCIA ONIRICA
}

\section{MENTAL HEALTH, NEGATIVE EXPERIENCES IN CHILHOOD, AND ITS IMPACT ON DREAM EXPERIENCES}

\author{
Alejandro Parra \\ Psicólogo. Instituto de Psicologia Paranormal. Buenos Aires, Argentina
}

ORCID: http://orcid.org/0000-0001-7943-2794

\section{Víctor Hugo Damián Silguero}

Facultad de Psicología y Relaciones Humanas

Universidad Abierta Interamericana. Buenos Aires, Argentina ORCID: https://orcid.org/0000-0002-7662-6005

Cómo referenciar este artículo/How to reference this article:

Parra, A. y Damián Silguero, V. H. (2020). Salud mental, experiencias negativas en la infancia y su impacto sobre la experiencia onirica. Revista de Psicoterapia, 31(117), 163-177. https://doi. org/10.33898/rdp.v31i117.435 


\title{
Resumen
}

Los sueños perturbadores son sueños vívidos caracterizados por emociones negativas intensas, y se encuentran entre los síntomas más frecuentes de las víctimas de trauma. Sus trastornos relacionados pueden persistir durante años e incluso décadas después del episodio traumático. El objetivo de este estudio fue evaluar si individuos que presentan más frecuentemente pesadillas o sueños de angustia han pasado por experiencias traumáticas en su infancia y su relación con rasgos psicopatológicos actuales. Se administraron tres instrumentos a una muestra de 446 adultos de la población general: el Cuestionario de Sueños, el Cuestionario de Experiencias Negativas en la Niñez; y una versión abreviada del Symptoms Assesment. El resultado muestra que los participantes presenta contenidos de sueños especificos, tales como a oír voces/ músicas en sueños (88\%), sueños lúcidos (79\%), terror nocturno (64\%), presencias malignas/demoníacas (61\%), y sueños que repiten un evento traumático (40\%). Los resultados también mostraron una correlación entre la frecuencia de experiencias oníricas y el maltrato parental (abuso emocional, sexual y físico) y una correlación positiva y significativa entre el recuerdo del sueño con propensión a síntomas de vulnerabilidad en salud mental, lo cual confirmó las dos hipótesis principales. Además, una diferencia de género mostró que los varones tendieron a tener mayor recuerdo del sueño en comparación con las mujeres, y las mujeres tendieron a mostrar sueños predominantemente más auditivos y terrores nocturnos en comparación con los varones.

Palabras claves: Pesadillas, Trauma infantil, Salud mental, Sueños perturbadores.

\begin{abstract}
Disturbing dreams are vivid dreams characterized by intense negative emotions, which are among the most frequent symptoms of trauma victims, and their related disorders can persist for years and even decades after the trauma. The aim is to evaluate whether individuals who most dream recall, nightmares or bad dreams, score higher on traumatic experiences in their childhood and if they present current disturbing symptoms. Three measures were used over a sample of 446 adults from the general population: The Dream Questionnaire, Child Negative Experiences Questionnaire; and shortened version of the Symptoms Assessment (SA-45). Descriptive results showed specific dream content, such as hearing voices/music in dreams (88\%), lucid dreams (79\%), nocturnal terror (64\%), evil entities (61\%), and recurrent disturbing dreams (40\%). The results also showed a correlation between the frequency of dream recall and higher scores of negative experiences in childhood, particularly parental abuse (emotional, sexual and physical) and a negative correlation between the dream recall and mental health. In addition, it confirmed the two main hypotheses: negative symptoms could "modulate" in some way the negative dream experiences. Gender differences also were found, i.e. male tended to have higher dream recall compared to female, and female tended to show predominantly more auditory dreams and nocturnal terrors compared to female.

Keywords: Nightmare, Childhood trauma, Psychopathological symptoms, Disturbing Dreams.
\end{abstract}


El sueño y su función ha sido siempre un tema de interés para distintas culturas y a través de los siglos. Se conocen historias de larga data acerca del sueño; éstas datan de hace más de dos mil años cuyas fundamentaciones se atribuían a aspectos de tipo místico. Sin embargo, esto indicaba cierta atención sobre la experiencia onírica (para más información, ver Aquino-Cias y Alonso-López, 2006). Por ejemplo, la teoría de Clara Hill (Hill y Rochlen, 2006) considera a los sueños como experiencias que representan vivencias significativas y conflictos sin resolver que irrumpen en la atención de la persona que sueña. Asimismo postula que los sueños son únicos para cada individuo y por ende, no pueden ser interpretados a través como símbolos estandarizados. Como alternativa, propone el modelo "cognitivo-experiencial" el cual integra la teoría psicoanalítica de la interpretación del propio paciente y sus sentimientos con la psicología cognitiva, que estudia los esquemas de pensamiento que se forman y que son almacenados por la memoria (Hill y Rochlen, 2006)

Un tipo de sueño perturbador conocido popularmente bajo el nombre de "pesadilla" puede estar asociado con la psicopatología, en particular, en adultos jóvenes (López Romera, 2006). Navarro Egea (2006) las define como: "sueños intensos y de temor, que pueden despertar dejando una sensación aguda de miedo y ansiedad, proyectando casi siempre trastornos o conflictos emocionales", y el 50\% de los adultos tuvieron pesadillas en algún momento de su vida (López Romera, 2006). Parra (2006) diferencia a las "pesadillas" respecto de los "terrores nocturnos" considerando que las primeras se producen en una fase REM del sueño (tono muscular nulo), mientras que los últimos son trastornos propios del sueño no-REM. Las pesadillas recurrentes se caracterizan por una alta comorbilidad del estado de ánimo y el trastorno de ansiedad. En el Trastorno Depresivo Mayor, las pesadillas a veces ocurren durante una enfermedad y disminuyen drásticamente durante el período de tratamiento. En otro estudio, se encontró una asociación significativa entre los sueños repetitivos y atemorizantes y la tendencia suicida en pacientes con depresión mayor (Agargun et al., 1998).

Miró-Morales y Martínez-Narváez (2004) sostienen que las pesadillas suelen aparecer pasada la segunda mitad de la noche, cuando la fase REM es más duradera y genera un malestar subjetivo que puede producir deterioros en diferentes áreas de la persona que lo padece, debido a que pueden propiciar la presencia de niveles altos de ansiedad, miedos diversos, sentimientos de indefensión y un empeoramiento en la calidad del sueño. Por otro lado, las personas que padecen pesadillas pueden presentar un deterioro en su funcionamiento diurno a causa de la angustia que puede estar asociada al recuerdo del sueño, el cansancio físico debido a la interrupción en el ciclo del sueño, y a las preocupaciones que se generan acerca del propio estado de la salud mental.

Otros autores, como Kramer y Kinney (1988), han sugerido, en contraposición a la clásica concepción de las pesadillas como fenómeno psicopatológico, que las mismas podrían funcionar como un mecanismo de afrontamiento frente al estrés. Los estresores podrían incorporarse a los sueños para de esta forma facilitar su afronta- 
miento. De esta forma las pesadillas cumplen una función adaptativa debido a que reducen el impacto del estresor mediante la repetida exposición al mismo a través de los sueños y, por otro lado, proporcionan una escena que podría ser aterradora para el soñante pero que resulta inofensiva y en la cual, se pueden contemplar posibles soluciones para el evento estresante (Miró-Morales y Martínez-Narváez, 2004).

Hartmann $(1996,1999)$ plantea que las personas que sufren de alta frecuencia de pesadillas se caracterizan por presentar cierta dificultad para regular de manera eficiente sus emociones (Miró-Morales y Martínez-Narváez, 2004) a causa de su limite "fino" (thin boundaries) el cual plantea que las pesadillas podrían reflejar una hipersensibilidad a los estímulos negativos debido a un bajo umbral de tolerancia a la activación emocional. Estudiando las conexiones que se dan entre el contenido de los sueños y las emociones, y luego de recopilar una larga serie de sueños de distintas personas que habían experimentado un trauma agudo, Hartmann (1996) descubrió como, por ejemplo, personas que escaparon de un incendio, víctimas de violación o presenciaron un asesinato, por lo general muestran claras conexiones entre el evento traumático y recuerdos del pasado. Estas conexiones parecen estar guiadas principalmente por preocupaciones emocionales que afectan al soñador. El proceso de conectar el trauma con otro material vinculado a las emociones del soñador (imaginación, lectura, etc.) se expande de forma gradual y absorbe cada vez más material. De esta forma el trauma juega un papel cada vez más pequeño en la vida del soñador $y$, por lo tanto, los sueños vuelven a su estadio previo al trauma (Hartmann, 1996).

Los sueños perturbadores son sueños vívidos caracterizados por emociones negativas intensas como miedo, ansiedad e ira (Levin y Nielsen, 2007; Zadra et al., 2006). Los sueños perturbadores incluyen pesadillas y sueños de angustia, y se encuentran entre los síntomas más frecuentes de las víctimas de trauma y los trastornos relacionados con los sueños. Pueden persistir durante años e incluso décadas después del trauma (Mellman y Hipólito, 2006; Schreuder et al., 2000). La frecuencia con la que se experimentan los trastornos del sueño relacionados con el trauma a lo largo del tiempo puede variar en función de la gravedad del trauma, el grado de exposición al trauma y el peligro asociado (Duval y Zadra, 2010; Wood et al., 1992). El abuso infantil constituye uno de los traumas crónicos mejor documentados. Su impacto en las variables conductuales, psicológicas y sociales puede aparecer a lo largo del desarrollo del individuo (Cicchetti y Lynch, 1995; Margolin y Gordis, 2000), y los niños que son víctimas de abuso y negligencia corren mayor riesgo de desarrollar psicopatología y angustia psicológica en la vida adulta (Banyard et al., 2001).

Agargun et al. (1998) observaron que los pacientes con diagnóstico de depresión mayor presentaban pesadillas frecuentes como sintomatología y tenían mayor probabilidad de presentar riesgo de suicidio, especialmente las mujeres. También se ha encontrado una asociación de las pesadillas a distintos tipos de trastorno psicótico, por ejemplo, un nivel significativo entre la frecuencia de pesadillas y 
trastornos límite de la personalidad y esquizotípico (Hartmann et al, 1981). Otros autores (Bearden, 1994) consideran que existe cierta similitud entre las pesadillas y los trastornos psicóticos como la esquizofrenia. Las pesadillas podrían representar una transición entre la normalidad y el trastorno psicótico a nivel onírico. Por otra parte la importancia del diagnóstico y tratamiento temprano para las pesadillas podría servir para detectar y prevenir posibles episodios psicóticos (Miró-Morales y Martínez-Narváez, 2004).

Sin embargo, pocos estudios han investigado la frecuencia y los correlatos de los sueños perturbadores adultos y su relación con el trauma en la infancia. De hecho, la frecuencia de los sueños perturbadores y la vigilia que generan los sueños de angustia y las pesadillas (reacciones de inquietudes del soñador durante las horas de vigilia sobre la experiencia del sueño perturbador) están moderadamente correlacionadas; y el estrés predice la psicopatología en la vida de vigilia (e.g., Belicki, 1992a; Blagrove et al., 2004; Levin y Nielsen, 2007). La angustia relacionada con los sueños perturbadores puede reflejar una personalidad caracterizada por angustia emocional reactiva aumentada (Levin y Nielsen, 2007) y posiblemente mediatiza la relación entre la frecuencia de sueños perturbadores y la psicopatología.

Por definición, las pesadillas son sueños aterradores que despiertan a un soñador al soñar (Zadra et al., 2006) o, más precisamente, al estado del movimiento ocular rápido (REM) (Nielsen y Levin, 2007). La pesadilla y los sueños de angustia están en el mismo continuo, pero el primero se puede memorizar y recordar claramente al despertar debido a su intensidad distintiva de la emoción (Schredl, 2003; 2006; Levin y Nielsen, 2007), características que provocan miedo (Zadra et al., 2006) y la asociación con síntomas psicopatológicos (Zadra y Doneri, 2000). Ha habido una amplia gama de estudios sobre la prevalencia de pesadillas, en términos de diferentes métodos de investigación, poblaciones, entornos e incluso tipos de pesadilla. La prevalencia de pesadillas en la población clínica varía de una vez al mes (30\% a 50\%) a una vez a la semana (10\% a 30\%) (Levin, 1994 ; Schredl, 2003; Zadra y Doneri, 2000).

Sin duda, además de relacionarse con la ansiedad y la angustia, las pesadillas se han asociado con un amplio espectro de trastornos psicológicos y rasgos negativos en relación con las psicopatologías, como el trastorno de estrés postraumático (TEPT) (Ohayon et al., 1997), depresión (Levin, 1998), esquizofrenia (Berquier y Ashton, 1992; Levin y Fireman, 2002) y trastorno disociativo (Berquier y Ashton, 1992; Molina, 1996). En términos generales, las personas con problemas de salud mental pueden haberse predispuesto a la angustia y la ansiedad personal, lo que afecta significativamente su calidad de sueño y la vida cotidiana (Levin y Nielsen, 2009). Las pesadillas se convierten en el signo de sus condiciones mentales y, a su vez, intensifican sus niveles de estrés y ansiedad. Hartmann y Basile (2003) también notaron que las personas con experiencias disociativas en la vida de vigilia reportaban pesadillas más frecuentes e intensas en relación con la distorsión de imagen corporal y los pensamientos, que eran similares a los estados de disociación. 
En consecuencia, los profesionales de la salud deben estar al tanto de las personas que tienen una alta frecuencia de pesadillas con el fin de mejorar su calidad diagnóstica en la práctica clínica. La pregunta aquí es ¿en qué medida los eventos traumáticos en la infancia activan pesadillas y de qué modo las pesadillas potencian el desarrollo de psicopatologías en la vida adulta? El objetivo de este estudio es evaluar individuos en la población general que experimentan pesadillas y sueños de angustia (sueños perturbadores) en relación con experiencias traumáticas infantiles y su efecto sobre la salud mental presente.

Las hipótesis aquí son que (H1) se encontrará una correlación positiva y significativa entre las experiencias oníricas perturbadoras y el grado de maltrato infantil, en otras palabras, los traumas en la infancia producen mayor frecuencia de pesadillas en la vida adulta, (H2) se encontrará una correlación positiva y significativa entre los sueños (suma total de la frecuencia de experiencias oníricas) y el grado de indicadores de psicopatología, en otras palabras, individuos que puntúan alto en rasgos psicopatológicos tenderán a producir mayor frecuencia de pesadillas, y (H3) se encontrará una correlación positiva y significativa entre los traumas en la infancia y un aumento en el grado de psicopatología (medido con el SA-45) en individuos que presentan mayor frecuencia de sueños, en otras palabras, los traumas que se dan en la infancia aumentan el riesgo de padecer mayor número de síntomas psicopatológicos en soñadores frecuentes.

\section{Método}

\section{Participantes}

La muestra estuvo integrada por 446 casos de ambos sexos, 73 (16.4\%) varones y $373(83.6 \%)$ mujeres, cuyo rango etario era de 18 a 55 años (Media = $36.06, D T=9.56)$. La muestra fue reclutada a través de dos fuentes, $81(18 \%)$ casos en formato impreso (distribución de los cuestionarios entre julio a noviembre del 2017) y 365 (82\%) recibidas a través de una plataforma on line generada por Google Formularios ${ }^{\circledR}$ subida a la web. Para el formato impreso, se distribuyeron los tres instrumentos entregados en mano y se dieron instrucciones para completarlos. Ambos formatos (impreso y on line) incluyó un Consentimiento Informado. Debido a la naturaleza de las respuestas dadas, particularmente el Cuestionario de Experiencias Negativas en la Niñez, los datos fueron tratados con confidencialidad $\mathrm{y}$ anonimato en sus respuestas.

\section{Instrumentos}

El Cuestionario de Sueños -Forma A (Parra, 2006, 2014) evalúa 17 tipos de sueños usando la clasificación creada por van de Castle (1994) con una escala de respuesta de tipo likert con un rango de $0=$ Nunca a $3=$ Casi siempre. Para el presente estudio, se seleccionaron sólo seis preguntas de las 17 originales; tres preguntas que connotan sueños predominantemente "negativos” (presencia maligna/ 
demoníaca, sueños que repiten un evento traumático, y terrores nocturnos), dos que connotan sueños con un tono más positivo o benigno (Sueños Lúcidos y Oír Voces/ Música) y sueños que pueden tener connotaciones, tanto negativas como positivas (sueños recurrentes). Para el presente estudio, se obtuvo además un puntaje para obtener una puntuación total sumando las respuestas de la respuesta de cada tipo de sueño (Rango $=0$ a 18 , Media $=7.44 ; D T=2.79$ ). El cuestionario posee alta validez discriminante y el valor de confiabilidad con un Alfa de Cronbach $=.83$ (Parra, 2006).

Cuestionario de Experiencias Negativas en la Niñez (CTQ; Bernstein et al., 1994; Fink etal., 1995; Bernstein et al., 1997). Es un cuestionario auto-administrable de perfil retrospectivo, que mide el grado de abuso sufrido durante la infancia. Los factores que evalúa son tres tipos de Abuso (Físico, Emocional, y Sexual) y dos tipos de Abandono (Físico y Emocional), y una puntuación total (excepto eventos traumáticos como la muerte de un padre o una enfermedad grave). El cuestionario contiene 28 items que se responden con una escala Likert de cinco puntos $(0=$ Nunca, $1=$ Una vez, $2=A$ veces y $3=$ Casi siempre), que indican que a mayor puntuación aumenta el grado de maltrato infantil. Posee alta validez discriminante (Bernstein, et al., 1997) y el valor de confiabilidad obtuvo un Alfa de Cronbach = .93 (Parra y Ugarte, 2018).

Symptoms Assesment (SA-45; Sandin et al., 2008). Es un instrumento derivado del SCL-90 que evalúa síntomas psicopatológicos de 45 ítems con una escala de respuesta de tipo Likert de cinco puntos $(0=$ No le ha molestado nada a $4=L e$ han molestado mucho). Consiste en nueve escalas de 5 ítems cada una que evalúan las mismas dimensiones que el SCL-90: Somatización, Obsesión-Compulsión, Sensibilidad Interpersonal, Depresión, Ansiedad, Hostilidad, Ansiedad Fóbica, Ideación Paranoide y Psicoticismo. El coeficiente alfa de Cronbach para el SA-45 total para la presente muestra fue de .95 .

\section{Resultados}

Respecto a la frecuencia del recuerdo del sueño, casi la mitad indicó soñar “Casi todas las mañanas" a "Una o dos veces por semana" (ambas 65\%) y la nitidez de sus sueños mostró ser "muy a moderadamente” clara e intensa (79\%) (ver Tabla 1). 
Tabla 1. Frecuencia de Recuerdo y Grado de Nitidez del sueño

\begin{tabular}{lcc}
\hline Recuerdo & $\mathrm{N}$ & $\%$ \\
\hline Casi todas las mañanas & 94 & 21.1 \\
\hline Una o dos veces por semana & 198 & 44.4 \\
\hline Una o dos veces por mes & 83 & 18.6 \\
\hline Casi nunca & 71 & 15.9 \\
\hline Nitidez y Claridad & & \\
\hline Perfectamente clara e intensa como la realidad & 91 & 20.4 \\
\hline Moderadamente clara y vivida & 266 & 59.6 \\
\hline Poco clara y vivida & 66 & 14.8 \\
\hline Vaga y difusa & 22 & 4.9 \\
\hline Tan vaga y difusa que resulta imposible discernir & 1 & 0.2 \\
\hline
\end{tabular}

Respecto al tipo de sueños, el $88 \%$ indicó oír voces/músicas en sueños, el $61 \%$ experimentó presencias maligna/demoníaca en sueños, el $73 \%$ tuvo sueños recurrentes, el $79 \%$ sueños lúcidos, $40 \%$ sueños que repiten un evento traumático, y el $64 \%$ experimentó terrores nocturnos (ver Tabla 2).

Tabla 2. Descriptivos de las Experiencias Oníricas

\begin{tabular}{lcc}
\hline Oír voces/músicas en sueños & $N$ & $\%$ \\
\hline Nunca & 52 & 11.7 \\
\hline Una vez & 34 & 7.6 \\
\hline Algunas veces & 264 & 59.2 \\
\hline Casi siempre & 96 & 21.5 \\
\hline Presencia maligna/demoníaca en sueños & & \\
\hline Nunca & 172 & 38.6 \\
\hline Una vez & 102 & 22.9 \\
\hline Algunas veces & 165 & 37.0 \\
\hline Casi siempre & 7 & 1.6 \\
\hline Sueños recurrentes & & \\
\hline Nunca & 116 & 26.0 \\
\hline Una vez & 100 & 22.4 \\
\hline Algunas veces & 210 & 47.1 \\
\hline Casi siempre & 20 & 4.5 \\
\hline Sueños Lúcidos & & \\
\hline Nunca & 94 & 21.1 \\
\hline Una vez & 76 & 17.0 \\
\hline Algunas veces & 245 & 54.9 \\
\hline Casi siempre & 31 & 7.0 \\
\hline Sueños que repiten un evento traumático & & \\
\hline Nunca & 264 & 59.2 \\
\hline Una vez & 81 & 18.2 \\
\hline Algunas veces & 95 & 21.3 \\
\hline Casi siempre & 6 & 1.3 \\
\hline
\end{tabular}




\begin{tabular}{lcc}
\hline Oír voces/músicas en sueños & $N$ & $\%$ \\
\hline Nunca & 52 & 11.7 \\
\hline Una vez & 34 & 7.6 \\
\hline Algunas veces & 264 & 59.2 \\
\hline Casi siempre & 96 & 21.5 \\
\hline Terrores nocturnos & & \\
\hline Nunca & 161 & 36.1 \\
\hline Una vez & 94 & 21.1 \\
\hline Algunas veces & 180 & 40.4 \\
\hline Casi siempre & 11 & 2.5 \\
\hline
\end{tabular}

Se llevó a cabo un contraste de hipótesis sobre la normalidad de las variables mediante un análisis de Kolmogorov-Smirnov ( $n>100$ casos). A partir de los valores obtenidos, se asumió una distribución altamente asimétrica de las puntuaciones de los tres instrumentos. En consecuencia, se decidió emplear para los análisis estadísticos, el test de $U$ de Mann-Whittney para comparar grupos y Rs de Spearman para correlacionar las puntuaciones de las escalas.

La H1 predice que se encontraría una correlación positiva y significativa entre el Index de sueños (suma de la frecuencia de las experiencias oníricas) y el grado de maltrato infantil, lo cual se confirmó ( $R s=.23, p<.001)$. Se encontraron además correlaciones significativas en Abuso ( $R s=.22, p<.001$ ), pero no en Abandono con el Index de sueños, así como correlaciones con tipos de sueños, tales como Oír voces/músicas en sueños ( $R s=.09, p<.037)$, Presencia maligna/demoníaca ( $R s=.13, p=.004)$, Sueños Recurrentes $(R s=.16, p<.001)$, Sueños Lúcidos (Rs $=.14, p=.003)$, y Sueños que repiten un evento traumático $(R s=.13, p=.003)$, excepto Terrores nocturnos (ver Tabla 3 ).

Tabla 3. Correlación entre la frecuencia de Sueños con Maltrato Infantil

\begin{tabular}{|c|c|c|c|c|c|c|c|}
\hline Variables & $\begin{array}{c}\text { Oir } \\
\text { voces/ } \\
\text { musicas }\end{array}$ & $\begin{array}{c}\text { Presencia } \\
\text { maligna/ } \\
\text { demoníaca }\end{array}$ & Recurrentes & Lúcidos & $\begin{array}{c}\text { Repiten } \\
\text { Evento } \\
\text { traumático }\end{array}$ & $\begin{array}{c}\text { Terrores } \\
\text { nocturnos }\end{array}$ & Sueños \\
\hline Abuso Físico & .05 & .07 & .09 & .01 & .09 & .02 & $.11^{\star *}$ \\
\hline Abuso Emocional & .12 & $.12^{*}$ & $.14^{\star *}$ & $.15^{\star \star}$ & $.15^{\star \star \star}$ & .03 & $.22^{\star \star \star}$ \\
\hline Abuso Sexual & .06 & $.11^{\star}$ & .04 & .06 & .05 & .07 & $.12^{\star \star}$ \\
\hline Ab. Fisico & .02 & .02 & .06 & .07 & .01 & .03 & .08 \\
\hline Ab. Emocional & .02 & .005 & .07 & .02 & .05 & .03 & .05 \\
\hline F1.Abuso & $.12^{\star \star}$ & $.12^{\star \star}$ & $.16^{\star \star \star}$ & $.12^{\star \star \star}$ & $.14^{\star \star \star}$ & .03 & $.22^{\star \star \star}$ \\
\hline F2.Abandono & .006 & .02 & .08 & .05 & .02 & .03 & .07 \\
\hline Maltrato Infantil & $.09^{*}$ & $.13^{\star \star}$ & $.16^{\star \star \star}$ & $.14^{\star \star}$ & $.13^{\star \star}$ & .05 & $.23^{\star \star \star}$ \\
\hline
\end{tabular}

${ }^{*} p<.05 ;{ }^{* \star} p<.01 ;{ }^{* \star} p<.001$

Corrección Bonferroni $=p$ ajustada .03

La $\mathrm{H} 2$ predice que se encontraría una correlación positiva y significativa entre el Index de sueños (suma de la frecuencia de experiencias oníricas) y el grado de 
psicopatología (medida con el SA-45), lo cual se confirmó ( $R s=.22, p<.001)$. Se encontraron además correlaciones significativas en ocho de las 9 psicopatologías que mide el SA-45 (con una Media de $R s=.16$ y una $p<.001$ ), excepto Ansiedad Fóbica (ver Tabla 4).

Tabla 4. Correlación entre el Tipo de Sueño (Index) con Rasgos Psicopatológicos

\begin{tabular}{|c|c|c|c|c|c|c|c|}
\hline Variables & $\begin{array}{c}\text { Oir voces/ } \\
\text { Músicas }\end{array}$ & $\begin{array}{c}\text { Presencia } \\
\text { maligna/ } \\
\text { demoniaca }\end{array}$ & Recurrentes & Lúcidos & $\begin{array}{c}\text { Repiten } \\
\text { Evento } \\
\text { traumático }\end{array}$ & $\begin{array}{c}\text { Terrores } \\
\text { nocturnos }\end{array}$ & $\begin{array}{l}\text { Sueños } \\
\text { s (Total) }\end{array}$ \\
\hline 1.Hostilidad & $.12^{\star \star}$ & $.10^{\star}$ & .06 & .02 & $.11^{*}$ & $.21^{\star \star \star}$ & $.20 \star \star \star$ \\
\hline 2.Somatización & $.12^{* *}$ & .05 & .08 & .04 & $.13^{\star *}$ & $.20^{\star \star \star}$ & $.19^{\star \star \star}$ \\
\hline 3.Depresión & .07 & $.11^{*}$ & .02 & .05 & $.17^{\star \star \star}$ & $.12^{\star \star \star}$ & $.18^{\star \star \star}$ \\
\hline 4.Obsesión/Compulsión & .07 & .09 & .07 & .03 & .11 & $.14^{\star \star}$ & $.18^{\star \star \star}$ \\
\hline 5.Ansiedad & $.14^{\star \star}$ & .07 & .02 & .06 & $.16^{\star \star \star}$ & $.14^{\star \star \star}$ & $.19^{\star \star \star}$ \\
\hline 6.Sensibilidad Interpersonal & -.02 & .06 & .06 & .07 & $.11^{\star \star}$ & $.12^{\star \star \star}$ & $.14^{\star \star \star}$ \\
\hline 7.Ansiedad Fóbica & .04 & -.02 & -.019 & -.03 & $.10^{\star \star}$ & $.16^{\star \star \star}$ & .08 \\
\hline 8.Ideación Paranoide & .06 & .12 & .05 & -.00 & $.17^{\star \star \star}$ & $.13^{\star \star \star}$ & $.18^{\star \star \star}$ \\
\hline 9.Psicoticismo & -.01 & .06 & .089 & .07 & $.17^{\star \star \star}$ & $.16^{\star \star \star}$ & $.18^{\star \star \star}$ \\
\hline Psicopatología & $.09 *$ & $.10^{*}$ & .06 & .05 & $.17^{\star \star *}$ & $.19 * \star \star$ & $.22^{\star *}$ \\
\hline
\end{tabular}

${ }^{*} p<.05 ;{ }^{* *} p<.01 ;{ }^{* \star *} p<001$

Corrección Bonferroni $=p$ ajustada .03

Debido al bajo número de no-soñadores ( $n=2)$, se decidió dividir por la Mediana $(\mathrm{Mn}=7)$ el total de la frecuencia de sueños (Rango $=0$ a 16) para obtener dos grupos de soñadores: Baja Frecuencia de sueño (BFS $n=167,37,4 \%$ ) y Alta Frecuencia de sueño (AFS $n=279,62,6 \%$ ). La H3 predice que se encontraría una correlación positiva y significativa entre el trauma en la infancia y un aumento en el grado de psicopatología(medida con el SA-45) en individuos que presentan mayor frecuencia de sueños, lo cual se confirmó ( $R s=.41, p<.001$ ), si bien -en rigor-el grupo BFS también presentó una correlación positiva y significativa, pero es menos robusta en comparación con el grupo de AFS ( $R s=.21, p=.005)$ (ver Tabla 5).

Tabla 5. Correlación entre Maltrato Infantil y Rasgos Psicopatológicos en Soñadores con Baja/Alta Frecuencia de Sueños

\begin{tabular}{lcc}
\hline & \multicolumn{2}{c}{$\begin{array}{c}\text { Psicopatología } \\
\text { (SA-45) }\end{array}$} \\
\hline F1.Abuso & $\begin{array}{c}\text { Baja Frecuencia } \\
\text { Sueños }(n=167)\end{array}$ & $\begin{array}{c}\text { Alta Frecuencia } \\
\text { Sueños }(n=279)\end{array}$ \\
\hline F2.Abandono & $.24^{\star \star}$ & $.39^{\star \star \star}$ \\
\hline Maltrato Infantil & .075 & $.35^{\star \star \star}$ \\
\hline${ }^{*} p<.05 ;{ }^{\star \star} p<.01 ;{ }^{* \star \star} p<001$ & $.21^{\star \star}$ & $.41^{\star \star \star}$ \\
Corrección Bonferroni $=p$ ajustada .03 & & \\
1.Baja Frecuencia $=0$ a 6 Alta Frecuencia $=7$ a 16 (Mediana $=7)$ &
\end{tabular}


Se llevaron a cabo análisis post-hoc con algunas variables demográficas tales como diferencias de Género, y Estado Civil, y correlaciones con la Edad. Para diferencias de género, se encontró que los varones tendieron a puntuar alto en Recuerdo de sueños $(z=2,25, p=.024)$ en tanto que las Mujeres tendieron a experimentar sueños de Oír Voces/Músicas $(z=2,43, p=.015)$ y Terrores nocturnos $(z=1,13$; $p=.032$ ) (ver Tabla 7), y se encontraron correlaciones negativas para la Edad con Abuso (Rs = -.09; $p=.042$ ) y con Psicopatología ( $R s=-.26, p<.001)$, pero no se encontraron diferencias significativas en Estado Civil.

\section{Discusión}

El objetivo del presente estudio fue evaluar si individuos que presentan más frecuentemente pesadillas o sueños de angustia han pasado por experiencias traumáticas en su infancia y presentan rasgos psicopatológicos actuales. Más específicamente, evaluar rasgos (o tendencias) psicopatológicas que se presentan con mayor frecuencia en personas que experimentan pesadillas, y su relación con el trauma infantil. Los resultados mostraron que los participantes presentan una tendencia predominante a oírvoces/músicas en sueños (88\%), tener sueños lúcidos (79\%), experimentar terrores nocturnos (64\%), y presencias malignas/demoníacas en sueños (61\%), y sueños que repiten un evento traumático (40\%). Además, el $65 \%$ indicó tener sueños con bastante frecuencia (Casi todas las mañanas a Una o dos veces por semana) y una mayor claridad y nitidez en sus sueños (79\%).

\section{Relación entre las Experiencias Oníricas y el Maltrato Infantil}

En primer lugar, los resultados mostraron una relación entre la frecuencia de experiencias oníricas y el grado de maltrato infantil, con un énfasis en el factor Abuso, donde el Abuso Emocional (e.g., "Mi familia abusaba de mi emocionalmente, por ejemplo, me insultaba, me humillaba, o me menospreciaba"), el sexual (e.g., “Me manosearon”), y el Físico (e.g., “Alguien en mi familia me castigo tan fuerte que tuve que ver a un médico"), demuestra que las experiencias negativas infantiles tienden a mostrar cierta "causalidad" con experiencias oníricas perturbadoras actuales, particularmente pesadillas, como presencias malignas y sueños que repiten situaciones traumáticas. El sueño recurrente mostró una correlación más robusta, lo cual podría indicar que la recurrencia (y persistencia) del sueño -incluso si repite experiencias traumáticas o si resultan perturbadores- podría ser el equivalente funcional de una experiencia nocturna que contribuye a "liberar" tensión psíquica acumulada, lo cual confirma los hallazgos de Hartmann y Basile (2006) que sugieren que estos sueños son más frecuentes en individuos que han pasado por eventos negativos (abusos) contribuyendo a descomprimir la tensión psíquica que les da origen.

En otras palabras, estos sueños bien pueden ser terapéuticos per se, y tienen gran valor en la vida emocional del soñante que experimentó abuso infantil. Sin embargo, esta misma relación no se presentó para situaciones de Abandono, tanto 
Físico como Emocional, lo cual el abandono podría tener una valencia diferente respecto a la situación de abuso. Posiblemente, las situaciones de abandono infantil -aunque claramente traumáticas- puedan ser de algún modo compensadas por las fortalezas y resiliencia de algunos individuos. Por otra parte, la experiencia de Terror nocturno, definida como el "despertar abrupto, sensación de amenaza, riesgo físico, o angustia pero sin poder recordar el contenido del sueño”, podrían no connotar como pesadilla en sentido estricto, o bien puede ser resultado de otros procesos independientemente de situaciones de trauma infantil, como el estrés (Blagrove et al., 2004; Levin et al., 2009; Zadra y Donderi, 2000).

\section{Relación entre las Experiencias Oníricas y Rasgos Psicopatológicos}

En segundo lugar, los resultados también mostraron una correlación positiva y significativa entre la frecuencia de experiencias oníricas (Index) con los rasgos psicopatológicos, donde casi todos los rasgos (excepto Ansiedad Fóbica) tendieron a mostrar relaciones positivas. Sin embargo, aunque las correlaciones son débiles (Rango de $R s=.06-.19$, Media $=.07$ ) debido a la alta asimetría del tamaño de la muestra, los sueños que repiten un evento traumático y "terrores nocturnos" tendieron a mostrar indicadores de psicopatología, seguido por "presencias malignas", y en menor grado "oír música/voces”.

Esto posiblemente debido a dos posibles causas: la primera, que los rasgos psicopatológicos puedan "moldear" de algún modo a las experiencias oníricas negativas, por ejemplo, individuos depresivos tienden a experimentar sueños de “caídas” (Parra, 2006) o “trituración de dientes”; individuos fóbicos o con ideación paranoide presentan más sueños asociados a presencia malignas (Levin y Nielsen, 2007), e individuos que presentan mayor somatización tienden a presentar sueños que repiten eventos traumáticos (Noll et al., 2006). De modo que es coherente sostener que los rasgos -o incluso cuadros clínicos específicos- presentan un patrón común de experiencias oníricas, incluso muchas de éstas con rico lenguaje simbólico en términos de su potencial interpretación dinámica. Segundo, los individuos que experimentan tales sueños son más vulnerables -o están en mayor riesgo- a experimentar perturbación psicológica, de hecho, los sueños que repiten eventos traumáticos y terrores nocturnos estuvieron asociados a rasgos tales como hostilidad (e.g., discusiones frecuentes), tendencia a la somatización (e.g., dolores musculares), depresión (e.g., sentirse desesperanzado respecto al futuro), obsesión (por ej. tener que comprobar una y otra vez todo lo que hace), ansiedad fóbica (e.g., evitar ciertos lugares o situaciones porque le dan miedo), y psicoticismo (e.g., los demás se dan cuenta de sus pensamientos).

Es notable que los sueños recurrentes -aun si son negativos-como los sueños lúcidos, no mostraron ningún indicador asociado a rasgos psicopatológicos, teniendo en cuenta que los sueños lúcidos tienden a ser interpretados como experiencias más positivas que perturbadoras. De hecho, los sueños traumáticos recurrentes también ocurren en pacientes con trastorno de estrés agudo, por lo tanto, las pesadillas 
parecen ser un mecanismo de afrontamiento eficaz (a cierto nivel inconsciente) en las víctimas del trauma. Los eventos traumáticos infantiles pueden jugar un papel clave en esta asociación (Wood y Bootzin, 1990).

Finalmente, los análisis post-hoc con variables demográficas, como diferencias de género, edad y estado civil, no mostraron resultados relevantes, excepto que los varones tienden a tener mayor recuerdo del sueño en comparación con las mujeres, y que las mujeres tendieron a mostrar sueños predominantemente más auditivos (oír voces/músicas) y terrores nocturnos en comparación con los varones. Respecto a otras variables, los más jóvenes tendieron a reportar situaciones de abuso y psicopatología, pero no tendencia a mayor pesadillas, y no se encontraron diferencias alguna en la frecuencia del soñar o la nitidez del sueño.

Este estudio permite inferir al menos dos potenciales predicciones para futuros estudios: En primer lugar, que la actividad onírica puede ser una variable que "modula" la posible psicogénesis de perturbación mental asociado a situaciones de abuso en la niñez, que puede convertirse en un claro cuadro clínico en la vida adulta. De hecho, un análisis de regresión logística lineal mostró que el maltrato infantil predice un modelo de rasgos psicopatológicos, lo cual confirma que las situaciones de maltrato, particularmente situaciones de abuso físico, emocional y sexual en la infancia podrían "actualizarse” en la vida adulta del soñante. En segundo lugar, una pregunta que emerge de este modelo es determinar si la experiencia onírica-que es interpretada por el sujeto como una "pesadilla" con connotaciones emocionalmente negativas- surge como una respuesta pre-consciente más bien adaptativa en lugar de disfuncional, que ayuda a afrontar el efecto perturbador o traumático de la experiencia negativa infantil que resulta luego en psicopatología en la vida adulta.

\section{Conclusiones}

Una posible limitación es la necesidad de tener más datos para confirmar estas especulaciones, como la edad infantil en que ocurrieron tales abusos, y un análisis más cualitativo (narrativo) de la experiencia onírica, lo cual excede la pretensión del objetivo planteado aquí. Otra limitación es el rango etario de la muestra, con énfasis en individuos más bien jóvenes (no mayores de 25/30 años) en lugar de una muestra más representativa del rango etario de la población general (cercano a una Media = 43 años), así como el número de individuos de la muestra que respondió la encuesta por internet ( $n=367,81 \%)$, produciendo un posible sesgo sencillamente porque están más interesados en buscar significado a sus sueños que la población general. Sin embargo, para responder a esta limitación se compararonambas respuestas pero no se encontraron diferencias significativas en maltrato infantil, ni frecuencia de sueños ni tampoco los rasgos psicopatológicos entre ambos grupos, tanto cuestionarios on line como impresos.

Una limitación final es la connotación negativa/positiva del sueño del presente estudio. En efecto, asumimos aquí que, por ejemplo, los sueños recurrentes 
tienden a ser "negativos" por su carácter repitente o que los sueños lúcidos son más "benignos" que perturbadores (Parra, 2006), pero no tenemos total certeza de ello a menos que tengamos las narrativas de los soñantes o una medida de su efecto benigno o perturbador, lo cual podría ser de gran interés para analizar más introspectivamente su naturaleza asociada a la evolución de cuadros clínicos y las consecuencias de los eventos negativos en la niñez.

Futuros estudios deberían testear la "hipótesis de la continuidad” ampliamente aceptada como una explicación convincente de por qué las personas con antecedentes psiquiátricos o rasgos psicopatológicos relevantes tienen pesadillas. Quizá los sentimientos emocionales o las experiencias disociativas en la vida de vigilia son simplemente una forma de continuidad en el sueño (Schredl, 2003, 2006). Además, las pesadillas son consideradas como un reflejo de las experiencias negativas reales en la vida diaria (Gabel, 1989, 1990). En realidad, el mecanismo explicado en el pasaje de la disociación es exactamente la esencia de la "hipótesis de la continuidad.”

\section{Referencias}

Agargun, M. Y., Çilli, A. S., Kara, H., Tarhan, N., Kinci, F. y Öz, H. (1998). Repetitive and frightening dreams and suicidal behavior in patients with major depression. Comprehensive Psychiatry, 39(4), 98-202. https:// doi.org/10.1016/S0010-440X(98)90060-8

Banyard, V. L., Williams, L. M. y Siegel, J. A. (2001). The long-term mental health consequences of child sexual abuse: An exploratory study of the impact of multiple traumas in a sample of women. Journal of Trauma \& Stress, 14, 697-715. https://doi.org/10.1023/A:1013085904337

Belicki, K. (1992). Nightmare frequency versus nightmare distress: Relations to psychopathology and cognitive style. Journal of Abnormal Psychology, 101(3), 592-597. https://doi.org/10.1037/0021-843X.101.3.592

Bernstein, D. P., Ahluvalia, T., Pogge, D. y Handelsman, L. (1997). Validity of the Childhood Trauma Questionnaire in an adolescent psychiatric population. Journal of the American Academy of Child \& Adolescent Psychiatry, 36(3), 340-348. https://doi.org/10.1097/00004583-199703000-00012

Bernstein, D. P., Fink, L., Handelsman, L. y Foote, J. (1994). Initial reliability and validity of a new retrospective measure of child abuse and neglect. American Journal of Psychiatry, 151(8), 1132-1136. https://doi. org/10.1176/ajp.151.8.1132

Berquier, A. y Ashton, R. (1992). Characteristics of the frequent nightmare sufferer. Journal of Abnormal Psychology, 101(2), 246-250.

Cicchetti, D. y Lynch, M. (1995). Failures in the expectable environment and their impact on individual development: The case of child maltreatment. En D. Cicchetti y D. J. Cohen (Eds.), Wiley series on personality processes. Developmental psychopathology, Vol. 2.: Risk, disorder, and adaptation (pp. 32-71). John Wiley \& Sons.

Duval, M. y Zadra, A. (2010). Frequency and content of dreams associated with trauma. Sleep and Medical Clinic, 5, 249-260. https://doi.org/10.1016/j.jsmc.2010.01.003

Fink, L. A., Bernstein, D., Handelsman, L., Foote, J. y Lovejoy, M. (1995). Initial reliability and validity of the Childhood Trauma Interview: Anew multidimensional measure of childhood interpersonal trauma. American Journal of Psychiatry, 152(9), 1329-1335. https://doi.org/10.1176/ajp.152.9.1329

Hartmann, E. (1996). Outline for a theory on the nature and functions of dreaming. Dreaming, 6(2), 147-170. https://doi.org/10.1037/h0094452

Hartmann, E. (1999). The nightmare is the most useful dream. Sleep Hypnosis, 1, 199-203.

Hartmann, E. y Basile, R. (2003). Dream imagery becomes more intense after 9/11. Dreaming, 13(2), 61-66. https://doi.org/10.1023/A:1023398924124

Hill, C. E. y Rochlen, A. B. (2006). El lenguaje de la noche. RIL Editores.

Kramer, M., Schoen, L. S. y Kinney, L. (1984). Psychological and behavioral features of disturbed dreamers. Psychiatric Journal of University of Ottawa, 9(3), 102-106. 
Levin, R. (1998). Nightmares and schizotypy. Psychiatry, 61(3), 206-216. https://doi.org/10.1080/00332747.1 998.11024832

Levin, R. y Fireman, G. (2002). Nightmare prevalence, nightmare distress, and self-reported psychological disturbances. Sleep, 25(2), 205-211. https://doi.org/10.1093/sleep/25.2.205

Levin, R., Lantz, E., Fireman, G. y Spendlove, S. (2009). The relationship between disturbed dreaming and somatic distress: A prospective investigation. Journal of Nervous and Mental Disease, 197(8), 606-612. https:// doi.org/10.1097/NMD.0b013e3181b0bd65

Levin, R. y Nielsen, T. (2007). Disturbed dreaming, posttraumatic stress disorder, and affect distress: A review and neurocognitive model. Psychological Bulletin, 133(3), 482-528. https://doi.org/10.1037/0033-2909.133.3.482

López Romera, A. (2006). Trastornos del sueño. En I. Suárez Richards (ed.), Introducción a la Psiquiatría (pp. 697-709). Polemos.

Margolin, G, y Gordis, E. B. (2000). The effects of family and community violence on children. Annals Review of Psychology, 51, 445-479. https://doi.org/10.1146/annurev.psych.51.1.445

Mellman, T. y Hipólito, M. M. S. (2006). Sleep disturbances in the aftermath of trauma and post-traumatic stress disorder. CNS Spectrum, 11(8), 611-615. https://doi.org/10.1017/S1092852900013663

Miró-Morales, E. y Martínez-Narváez, P. (2004). Tratamientos psicológicos de las pesadillas: una revisión. International Journal of Psychology and Psychological Therapy, 4(1).123-132.

Molina, J. M. (1996). Dissociation in dreams: The impact of dissociative tendency on dream content. The Sciences and Engineering, 57(6), 4076-4080.

Navarro Egea, J. (2006). Sueños y pesadillas. International Journal of Developmental and Educational Psychology, 3(1), 265-279.

Noll, J. G., Trickett, P. K., Susman, E. J. y Putnam, F. W. (2006). Sleep disturbances and childhood sexual abuse. Journal of Pediatric Psychology, 31, 469-480. https://doi.org/10.1093/jpepsy/jsj040

Ohayon, M. M., Moreslli, P. L. y Guilleminault, C. (1997). Prevalence of nightmares and their relationship to psychopathology and daytime functioning in insomnia subjects. Sleep, 20(5), 340-348. https://doi. org/10.1093/sleep/20.5.340

Parra, A. (2006). Sueños: Como interpretar sus mensajes. Kier.

Parra, A. (2009). El mundo oculto de los sueños. Kier.

Parra, A. (2014). Diferencias individuales en la frecuencia del recuerdo de sueños exóticos. Suma Psicológica, 21(1), 63-69. https://doi.org/10.1016/S0121-4381(14)70008-6

Parra, A. y Ugarte, M. (2018). Experiencias negativas en la niñez y estilo parental dominante en individuos que reportan experiencias paranormales. Perspectivas en Psicología, 15(1), 86-97.

Romans, S. E., Martin, J. L., Morries, E. y Herbison, G. P. (1999). Psychological defense styles in Women who report childhood sexual abuse: A controlled community study. American Journal of Psychiatry, 156(7), 1080-1085.

Schredl, M. (2003). Effects of state and trait factors on nightmare frequency. European Archives of Psychiatry Clinical Neuroscience, 253, 241-247. https://doi.org/10.1007/s00406-003-0438-1

Schredl, M. (2006). Factors affecting the continuity between waking and dreaming: Emotional intensity and emotional tone of the waking-life event. Sleep and Hypnosis, 8(1), 1-5.

Schreuder, B. J., Kleijn, W. C. y Rooijmans, H. G. (2000). Nocturnal re-experiencing more than forty years after war trauma. Journal of Trauma Stress, 13, 453-463. https://doi.org/10.1023/A:1007733324351

van de Castle, R. l. (1994). Our dreaming mind: A sweeping exploration of the role that dreams have played in politics, arts, religion, and psychology. Ballantine Books.

Wood, J. M. y Bootzin, R. R. (1990). The prevalence of nightmares and their independence from anxiety. Journal of Abnormal Psychology, 99(1), 64-68. https://psycnet.apa.org/doi/10.1037/0021-843X.99.1.64

Wood, J. M., Bootzin, R. R., Rosenhan, D., Nolen-Hoeksema, S. y Jourden, F. (1992). Effects of the 1989 San Francisco earthquake on frequency and content of nightmares. Journal of Abnormal Psychology, 101(2), 219-224. https://doi.org/10.1037/0021-843X.101.2.219

Zadra, A. y Doneri, D. C. (2000). Nightmares and bad dreams: Their prevalence and relationship to well-being. Journal of Abnormal Psychology, 109, 273-281.

Zadra, A., Pilon, M. y Donderi, D. C. (2006). Variety and intensity of emotions in nightmares and bad dreams. Journal of Nervous and Mental Disease, 194(4), 249-253. https://doi.org/10.1097/01.nmd.0000207359.46223.dc 